\title{
Detection of DNA strand breaks by fluroscence assisted DNA unwinding (FADU) assay in Hela cells treated with berbrine chloride before exposure to various doses of $\gamma$ - radition
}

\begin{abstract}
The DNA damage plays an important role in the cell death and DNA double strand breaks in particular have been implicated in cell mortality. Therefore, the ability of berberine chloride to modulate radiation-induced DNA damage in HeLa cells exposed to different doses of $\gamma$-rays was studied by fluorescence assisted DNA unwinding assay. The spontaneous frequency of undamaged DNA remained unaltered in $\mathrm{HeLa}$ cells treated with $0,1,2,4,6$ or $8 \mu \mathrm{g} / \mathrm{ml}$ of BCL at $0,0.25,0.5,1,2$ or $4 \mathrm{~h}$ postirradiation. Immediate exposure of HeLa cells to $3 \mathrm{~Gy} \gamma$-radiation (0h post-irradiation group) caused a drastic rise in the DNA strand breaks as evident by an abrupt reduction in the undamaged double stranded DNA (dsDNA). An elevation in the undamaged ds DNA was observed with time up to $12 \mathrm{~h}$ post-irradiation in cells exposed to $3 \mathrm{~Gy}$ irradiation without $\mathrm{BCL}$ treatment, indicating repair of radiation-induced DNA damage. However, incubation of HeLa cells with different concentrations of BCL before 3Gy irradiation caused a dose-dependent increase in the DNA strand breaks at all post-irradiation times. The highest DNA strand breaks were observed for $8 \mu \mathrm{g} / \mathrm{ml}$ $\mathrm{BCL}$ after exposure to $3 \mathrm{~Gy}$. The DNA strand breaks did not show repair up to $12 \mathrm{~h}$ in cells exposed $3 \mathrm{~Gy}$. The exposure of HeLa cells to 0.25 to $4 \mathrm{~Gy} \gamma$-radiation resulted in a dose dependent decline in dsDNA at all post-irradiation times and treatment of $\mathrm{HeLa}$ cells with $8 \mu \mathrm{g} / \mathrm{ml}$ berberine before irradiation led to a further attrition in the dsDNA. Our study demonstrates that irradiation of HeLa cells resulted in a dose dependent increase in DNA strand breaks and berberine treatment further increased the DNA strand breaks which may be one of the mechanisms of cell death.
\end{abstract}

Volume 3 Issue I - 2018

\author{
Ganesh Chandra Jagetia,' Shaival K. Rao² \\ 'Department of zoology, Mizoram University, India \\ ${ }^{2}$ Shree Samanvay Institute of Pharmaceutical Education and \\ Research, India
}

Correspondence: Ganesh Chandra Jagetia, Department of zoology, Mizoram University 10, Maharana Pratap Colony, Sector-13, Hiran Magri, Udaipur-3 I 3002, India, Email gc.jagetia@gmail.com

Received: November 03, 2017 | Published: January 29, 2018

Keywords: berberine, hela cells, DNA damage, FADU, irradiation

\section{Introduction}

Ionizing radiations are in frequent clinical use for diagnosis and treatment and the fact is that $50 \%$ of the patients visiting Cancer Treatment Centers for cancer therapy receive radiotherapy either alone or in conjunction with chemotherapy. ${ }^{1,2}$ The radiotherapy and chemotherapy both kills tumor cells by inflicting damage to cellular DNA and it is also known that the underlying cause of cancer is the induction of mutations into DNA by physical or chemical agents. ${ }^{3-5}$ The DNA damaging agents, including ionizing radiations and interstrand DNA cross-linking compounds have been used alone or as a combined treatment modality against cancer due to their extreme DNA damaging ability on proliferating cells. The efficacies of radiation therapy and chemotherapy as well as the mutagenic potential of the DNA damaging agents used in these therapies are modulated by the ability of cells to repair the inflicted DNA damage. ${ }^{6}$ Investigation of the molecular mechanisms of DNA damage repair and maintenance of genome stability and their biological effects are important. One of the most genotoxic DNA lesions that results from treatment with ionizing radiation or DNA cross linking compounds is the DNA double-strand break (DSB). DSBs are caused not only by exogenous sources but also by endogenous sources such as radicals generated during metabolic processes. ${ }^{4-5}$ In addition, a predominant source of DSBs in dividing cells is the process of DNA replication itself. Since DNA strand breaks play an important role in mutagenesis and oncogenesis, their dose- effect relationships and their rejoining kinetics are essential indicators that reflect cellular response processes. ${ }^{6-8}$ In addition to the primarily induced DNA breaks many other DNA lesions may be transformed into strand breaks by cellular repair processes and can thus easily be measured and quantified. ${ }^{4,5}$

The Fluorometric analysis of DNA unwinding (FADU) method has been reported to be as sensitive as the alkaline filter elution technique in detecting DNA strand breaks induced by X-rays or after treatment with radical-generating chemicals., ${ }^{911}$ FADU technique has been also useful in the identification of effects of occupational exposure to industrial air pollutants. ${ }^{11}$ Moreover, FADU assay is 3-4 times more sensitive than unscheduled DNA synthesis in detecting mutagenic effects In vitro by direct acting mutagens or environmental agents in various systems. ${ }^{12,13}$ The number of free DNA ends may be calculated after alkaline unwinding either from the size-distribution of unwound $\mathrm{DNA}^{9}$ or from the numerical discrimination between ssDNA and dsDNA. In this respect, fluorescent dyes are useful tools. FADU assay is a fast, sensitive and reliable method for the detection of strand breaks in DNA as an index of DNA damaging potential of radiation and/or chemical agents. ${ }^{13}$

The medicinal plants have a long history of use in the treatment of various ailments including cancer and many plants contain berberine, has formed the part of traditional systems including Ayurvedic and Chinese systems of medicine. Several clinically important medicinal 
plants, including Arcangelisia Flava, Berberis aquifolium (Oregon grape), Berberis aristata (tree turmeric), Hydrastis canadensis (goldenseal), Coptis chinensis (coptis or golden thread), Radix scutellariae and Tinospora cordifolia (giloy) have shown the presence of isoquinoline alkaloid, berberine. The antibacterial, antimicrobial, antioxidant, antidepressant, and anti inflammatory activities of berberine have been reported earlier. ${ }^{14-17}$ It has been also reported to be anti diarrheal, anti arrhythmic, antihypertensive, anti osteoarthritis, chemo sensitizing, hepatoprotective and neuro protective. ${ }^{18-20}$ Berberine treatment has been found to protect rats against ischemiareperfusion injury. ${ }^{21,22}$ Clinically berberine administration has been reported to reduce glucose, total cholesterol and low-density lipoprotein cholesterol levels in the diabetic patients. ${ }^{23-25}$ Clinical trials have indicated that chronic congestive heart failure patients treated with 1.2 to $2 \mathrm{~g}$ of berberine daily showed an improvement in the left ventricular ejection fraction and ventricular premature complexes. ${ }^{26}$ In yet another clinical trial berberine has been found to be effective in treating dementia dyslipidemia, hyperlipidemia, ocular Behcet's disease, and non-fatty liver disease. ${ }^{27-32}$ The anti angiogenic and anticancer activity of berberine has been reported in several studies. ${ }^{33-37}$ Beberine chloride has also been reported to increase the tumor radio sensitivity in irradiated HeLa cells. ${ }^{38}$ Berberine sulfate has been reported to significantly alleviate tumor yield in the 7,12-dimethylbenz $(a)$ anthracene (DMBA) induced tumors in rats. ${ }^{39}$ Therefore, the present study was undertaken to study the role of berberine chloride in enhancing the radiation-induced DNA damage in HeLa cells exposed to different doses of $\gamma$ - radiation by FADU assay.

\section{Materials and methods}

Minimum Essential Medium (MEM), Fetal calf serum (FCS), Hoechst 33258, try pan blue, sodium dodecyl sulphate (SDS), urea, $\beta$-mercaptoethanol, cyclohexane diamine tetra acetate (CDTA), were obtained from Sigma Chemical Company, St. Louis, USA, whereas rest of the chemicals were procured from Ranbaxy Fine Chemicals Limited, Mumbai, India.

\section{Preparation of drugs}

Doxorubicin hydrochloride (DOX) or Adrim, a kind gift from Dabur Pharmaceuticals, New Delhi, India and berberine chloride (BCL) were dissolved in sterile double distilled water (DDW) at a concentration of $5 \mathrm{mg} / \mathrm{ml}$ and diluted in MEM as required. All drug solutions were prepared afresh immediately before use. HeLa S3 cells having a doubling time of $20 \pm 2 \mathrm{~h}$ were procured from National Centre for Cell Science, Pune, India, and were used throughout the study. The cells were routinely grown in $25 \mathrm{~cm}^{2}$ culture flasks (Techno Plastic Products, Trasadingën, Switzerland) containing Eagle's minimum essential medium (MEM) supplemented with $10 \%$ fetal calf serum, $1 \%$ L-glutamine and $50 \mu \mathrm{g} / \mathrm{ml}$ gentamicin sulfate at $37^{\circ} \mathrm{C}$ in an atmosphere of $5 \% \mathrm{CO}_{2}$ in humidified air in a $\mathrm{CO}_{2}$ incubator (Nu Air, Plymouth, USA) with their caps loosened.

\section{Experimental design}

A fixed number $\left(5 \times 10^{5}\right)$ of exponentially growing cells were inoculated into several culture flasks (Techno Plastic Products, Trasadingën, Switzerland) and were allowed to complete two division cycles before the onset of experiments.

\section{Experiment I: Selection of optimum dose}

DDW + Irradiation: HeLa cells were treated with an equivalent amount of DDW before exposure to 3Gy $\gamma$-radiation.
DOX + Irradiation: This group of HeLa cells was treated with $2 \mu \mathrm{g} /$ $\mathrm{ml}$ DOX, before exposure to 3Gy $\gamma$-radiation.

BCL + Irradiation: The cell cultures of this group received 0, 1, 2, 4, 6 or $8 \mu \mathrm{g} / \mathrm{ml}$ BCL before exposure to 3Gy of $\gamma$-radiation.

DNA strand breaks in both groups were studied at $0,0.25,0.5,1,2$, 4,6 or $12 \mathrm{~h}$ post-irradiation.

\section{Experiment 2: Effect of $B C L$ on radiation-induced DNA strand breaks}

A separate experiment was performed to study the effect of $8 \mu \mathrm{g} / \mathrm{ml}$ $\mathrm{BCL}$ on the radiation-induced DNA damage, where groupings and other conditions were essentially similar to that described above except that HeLa cells were treated with $8 \mu \mathrm{g} / \mathrm{ml} \mathrm{BCL}$ and then exposed to $0,1,2,3$ or $4 \mathrm{~Gy} \gamma$-radiation. The DNA damage was evaluated at $0,0.25,0.5,1,2$ or $4 \mathrm{~h}$ post-irradiation.

\section{Irradiation}

After 4 hours of the above treatments, the cells were exposed to either 0 or $3 \mathrm{~Gy}$ (Experiment No. 1) or $0,0.5,1,2,3$ or $4 \mathrm{~Gy} \gamma$-radiation (Experiment No. 2) from a Tele cobalt therapy source (Theratron Atomic Energy Agency, Ontario, Canada) at a dose rate of $1 \mathrm{~Gy} / \mathrm{min}$ and at a distance (SSD) of $91 \mathrm{~cm}$.

\section{Fluorescence assisted DNA unwinding (FADU)}

The fluorescence assisted DNA unwinding (FADU) assay detects DNA single strand, double strand breaks and alkali labile sites (DNA breaks). The alteration in radiation-induced DNA breaks by BCL in HeLa cells was assessed using FADU assay in triplicate as described earlier with minor modifications. ${ }^{40}$ Briefly, the cells were incubated with $1 \mathrm{ml}$ of reagent mixture (containing $0.3 \mathrm{ml}$ of $6 \mathrm{~m} \mathrm{M}$ myoinositol, $0.1 \mathrm{ml}$ of $2 \%$ urea, $0.1 \mathrm{ml}$ of $5 \% \mathrm{SDS}, 0.4 \mathrm{ml}$ of $5 \mathrm{M} \mathrm{NaOH}$ and $0.1 \mathrm{ml}$ of $2 \mathrm{mM}$ CDTA in MilliQ water (Millipore, Billerica, MA, USA) for $1 \mathrm{~h}$ at $15^{\circ} \mathrm{C}$. The samples were flash frozen for $1 \mathrm{~min}$ and $0.4 \mathrm{ml}$ neutralizing mixture (containing $1 \mathrm{M}$ glucose, and $14 \mathrm{mM}$ beta mercaptoethanol), was added at room temperature. The samples were immediately sonicated for 5seconds (Sonics Vibra-cell, Newtown, CT, USA) to inhibit re association of complementary strands of DNA and to reduce the molecular weight of DNA. Each cell sample was transferred to a quartz curette and mixed gently with $1 \mathrm{ml}$ Hoechst $33258(5 \mu \mathrm{g} /$ $\mathrm{ml}$ ) by single inversion and the fluorescence was read at an excitation and emission wavelengths $350 \mathrm{~nm}$ and $450 \mathrm{~nm}$, respectively using a spectrofluorimeter (SFM 25, Kontron Instruments, Neufahrn, Germany) at room temperature.

The percent undamaged double stranded DNA and DNA strand breaks in the treatment groups were calculated as follows:

Undamaged double stranded DNA (\%)=(P-B/T-B) x 100

Percent DNA strand breaks $\mathrm{DSB}(\mathrm{s})=100$-percent undamaged double stranded DNA, where

P (Partial de maturation): HeLa cells, reagent mixture but without dye.

B: HeLa cells, DDW/BCL and dye but without reagent mixture.

T: HeLa cells, DDW/BCL, reagent mixture and dye.

The strand scission factor (SSF) is defined as $\ln \mathrm{F}_{\mathrm{t}} / \mathrm{F}_{\mathrm{t}=0}$ and was calculated as follows: $-\mathrm{SSF}=\log (\% \mathrm{dsDNA}$ in sample $/ \% \mathrm{dsDNA}$ in control). 


\section{Statistical analysis}

The statistical analyses were performed using Graph Pad Prism 6 statistical software (Graph Pad Software, San Diego, CA, USA). The significance among different treatments was determined by oneway ANOVA and Bonferroni's post-hoc test was applied for multiple comparisons. The experiments were repeated for confirmation of results. The results are the average of five individual experiments. The test of homogeneity was applied to find out variation among each experiment. The data of each experiment did not differ significantly from one another and hence, all the values have been combined and means calculated. A p value of $<0.05$ was considered statistically significant.

\section{Results}

The results are expressed as percent undamaged double stranded $\mathrm{DNA} \pm \mathrm{SEM}$ in tables 1-2 and (Figure 1) and strand scission factor \pm $\mathrm{SEM}(\mathrm{SSF} \pm \mathrm{SEM})$ in (Figure 2).



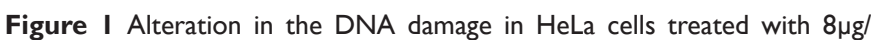
$\mathrm{ml}$ berberine chloride before exposure to different doses of $\gamma$-radiation at various post-irradiation times. Closed symbols, MEM+irradiation and Open symbols, BCL+ irradiation. Squares, Oh; Diamonds, 0.25h. Hexagons, 0.5h; Stars Ih, Pentagon, $2 \mathrm{~h}$ and circles, $4 \mathrm{~h}$ post-irradiation.

\section{Experiment I: Selection of optimum dose}

Treatment of HeLa cells with various concentrations of BCL did not alter the baseline frequency of DNA strand breaks as differences between the control and BCL treatment was non-significant except for 6 and $8 \mu \mathrm{g} / \mathrm{ml} \mathrm{BCL}$ (Table 1). Assessment of DNA damage in HeLa cells immediately after exposure to $3 \mathrm{~Gy}$ ( $0 \mathrm{~h}$ post-irradiation group) caused a drastic reduction in the undamaged double stranded DNA (dsDNA). An elevation in undamaged dsDNA was observed with time up to $12 \mathrm{~h}$ post-irradiation in cells exposed to $3 \mathrm{~Gy}$ irradiation without BCL treatment, indicating repair of radiation-induced DNA damage. Treatment of HeLa cells with different concentrations of BCL before 3 Gy irradiation caused a dose-dependent reduction in the undamaged dsDNA at all post-irradiation times. The greatest DNA damage was observed for $8 \mu \mathrm{g} / \mathrm{ml} \mathrm{BCL}$ after exposure to $3 \mathrm{~Gy}$. The maximum DNA strand breaks were observed at $4 \mathrm{~h}$ post-irradiation in HeLa cells treated with various concentrations of $\mathrm{BCL}$ before $3 \mathrm{~Gy}$ irradiation that remained almost unchanged up to $12 \mathrm{~h}$ post-irradiation after $3 \mathrm{~Gy}$ irradiation group (Table 1). When the evaluation of dsDNA was carried out at different post-treatment times giving allowance for repair of DNA, it was observed that the frequency of DNA strand breaks increased with the evaluation time for all doses of BCL in conjunction with 3Gy up to $12 \mathrm{~h}$ post-irradiation (Table 1). Treatment of HeLa cells with different concentrations of BCL before exposure to $3 \mathrm{~Gy}$ resulted in a BCL concentration dependent elevation in DNA strand breaks as evident by a constant decline in undamaged dsDNA with increasing concentration of BCL (Table 1). Treatment of HeLa cells with $4 \mu \mathrm{g} / \mathrm{ml}$ BCL before $3 \mathrm{~Gy}$ irradiation increased DNA strand breaks by approximately $50 \%$ when compared with concurrent non-drug treated $3 \mathrm{~Gy}$ irradiated cells at $4 \mathrm{~h}$ post-irradiation time. Therefore, further studies were undertaken using this concentration.

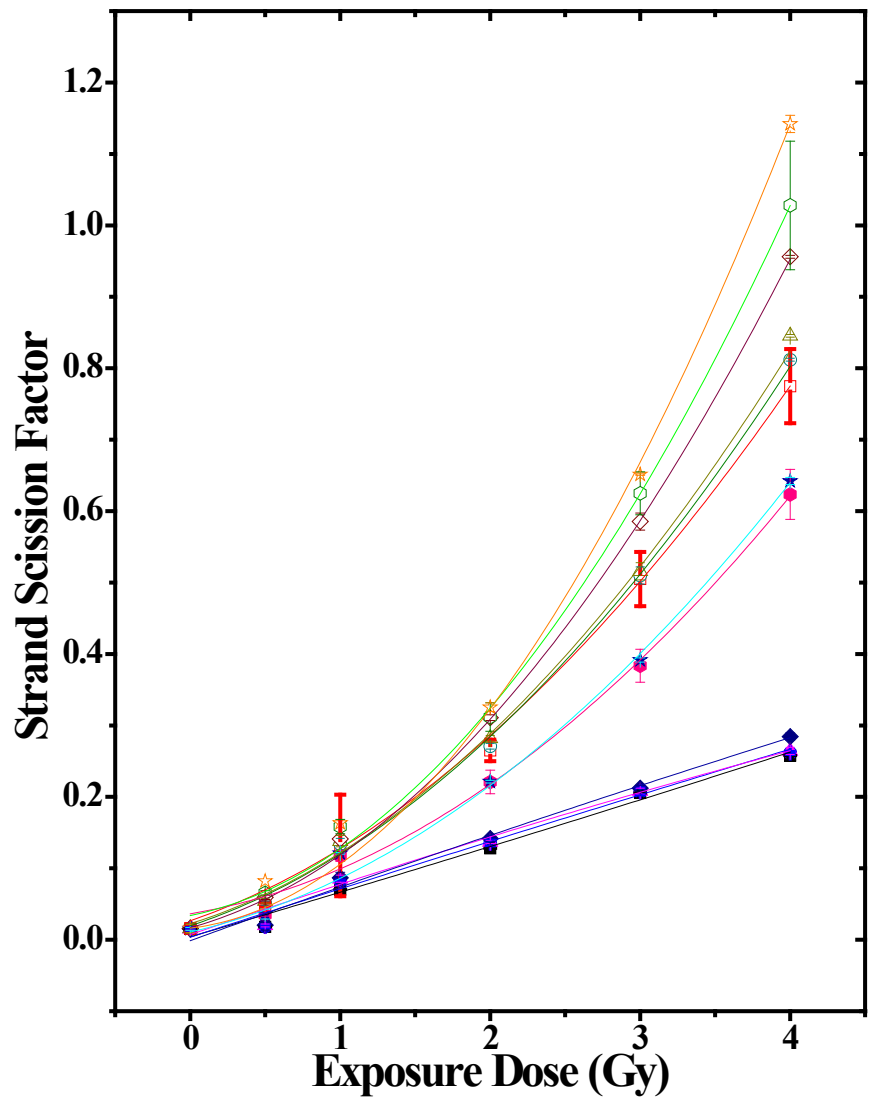

Figure 2 Alteration in the strand scission factor in HeLa cells treated with 8 $\mu \mathrm{g} / \mathrm{ml}$ berberine chloride before exposure to different doses of $\gamma$-radiation at various post-irradiation times. Closed symbols, DDW+irradiation; Open symbols, BCL+irradiation. Squares, $0 \mathrm{~h}$; Circles, 0.25h. Triangles, 0.5h; Diamonds, $\mathrm{Ih}$; Hexagon, $2 \mathrm{~h}$ and Stars, $4 \mathrm{~h}$ post-irradiation.

\section{Experiment 2: Effect of $B C L$ on radiation-induced DNA strand breaks}

Spontaneous DNA strand breaks in HeLa cells were approximately $2.5 \%$. Irradiation of HeLa cells to various doses of $\gamma$-radiation resulted in a dose-dependent increase in DNA strand breaks at all post-irradiation times and the highest strand breaks were estimated at $0 \mathrm{~h}$ post-irradiation for all exposure doses (Figure 1). A time dependent repair in DNA strand breaks was observed in the DDW + irradiation group, and a maximum 
repair was discernible at $4 \mathrm{~h}$ post-irradiation for $0.5 \mathrm{~Gy}$ irradiation, where only $10 \%$ of DNA was found to be damaged (Table 2 ). The repair of DNA strand breaks was minimal for $4 \mathrm{~Gy}$ even up to $4 \mathrm{~h}$ post-irradiation as $65 \%$ of the DNA still remained damaged (Table 2). Treatment of HeLa cells with $8 \mu \mathrm{g} / \mathrm{ml}$ of BCL before irradiation to various doses of $\gamma$-rays resulted in a significant elevation in the DNA strand breaks at all post-irradiation times. The cells exposed to $0.5 \mathrm{~Gy}$ and $1 \mathrm{~Gy}$ after BCL treatment showed repair of damaged DNA with time and a maximum repair of DNA was observed at $4 \mathrm{~h}$ post-irradiation (Table 2). However, treatment of HeLa cells with $8 \mu \mathrm{g} / \mathrm{ml} \mathrm{BCL}$ before exposure to $2-4 \mathrm{~Gy}$ resulted in a continuous elevation in damaged DNA up to $4 \mathrm{~h}$ post-irradiation where $95 \%$ of the DNA remained damaged after 4Gy exposure in BCL + irradiation group (Table 2).

Table I Effect of various concentrations of berberine chloride on the radiation-induced DNA strand breaks in HeLa cells exposed to 3 Gy $\gamma$ - radiation

\begin{tabular}{|c|c|c|c|c|c|c|c|c|c|c|c|c|c|c|}
\hline \multirow{4}{*}{$\begin{array}{l}\text { Post- } \\
\text { irradiation } \\
\text { time (h) }\end{array}$} & \multicolumn{14}{|c|}{$\begin{array}{l}\text { Undamaged } \\
\text { DNA (\%) } \pm \text { SEM }\end{array}$} \\
\hline & \multirow{2}{*}{\multicolumn{2}{|c|}{ DDW I $0 \mu \mathrm{l}$}} & \multirow{2}{*}{\multicolumn{2}{|c|}{ DOX $2 \mu g / m l$}} & \multicolumn{10}{|c|}{ Berberine chloride ( $\mu \mathrm{g} / \mathrm{ml})$} \\
\hline & & & & & \multicolumn{2}{|l|}{$\mathbf{I}$} & \multicolumn{2}{|l|}{2} & \multicolumn{2}{|l|}{4} & \multicolumn{2}{|l|}{6} & \multicolumn{2}{|l|}{8} \\
\hline & SIR & IR & SIR & IR & SIR & IR & SIR & IR & SIR & IR & SIR & IR & SIR & IR \\
\hline \multirow{2}{*}{0} & $98.49 \pm$ & $44.44 \pm$ & $97.12 \pm$ & $40.72 \pm$ & $98.24 \pm$ & $41.63 \pm$ & $97.58 \pm$ & $40.55 \pm$ & $96.57 \pm$ & $39.75 \pm$ & $95.18 \pm$ & $33.23 \pm$ & $94.89 \pm$ & $30.25 \pm$ \\
\hline & 2.71 & 1.10 & 2.63 & 0.88 & 2.46 & 0.96 & 2.22 & 0.78 & 2.04 & 0.74 & 2.03 & 0.71 & 2.04 & 1.24 \\
\hline \multirow{2}{*}{0.25} & $98.28 \pm$ & $44.6 \pm$ & $96.78 \pm$ & $39.22 \pm$ & $98.16 \pm$ & $40.34 \pm$ & $97.52 \pm$ & $39.12 \pm$ & $96.25 \pm$ & $39.48 \pm$ & $95.11 \pm$ & $33.12 \pm$ & $94.51 \pm$ & $29.84 \pm$ \\
\hline & 2.36 & 1.05 & 2.22 & $0.69 \approx$ & 2.18 & $1.13^{* \$}$ & 2.3 & $0.97^{\star \$}$ & 2.14 & $0.88^{\star \#}$ & 2.03 & 0.74 \#\# & 2.14 & $0.88^{\# \#}$ \\
\hline \multirow{2}{*}{0.5} & $98.17 \pm$ & $45.54 \pm$ & $96.47 \pm$ & $37.64 \pm$ & $97.88 \pm$ & $39.43 \pm$ & $97.38 \pm$ & $36.52 \pm$ & $95.86 \pm$ & $36.22 \pm$ & $95.02 \pm$ & $31.05 \pm$ & $94.24 \pm$ & $27.29 \pm$ \\
\hline & 2.48 & 1.16 & 2.40 & $0.71 * \$$ & 2.2 & $0.86^{\star \$}$ & 2.24 & $0.72^{* \$}$ & 2.17 & $0.61 * \#$ & 2.14 & $0.84 * \#$ & 2.34 & 0.84 \\
\hline \multirow{2}{*}{ I } & $98.14 \pm$ & $46.52 \pm$ & $96.11 \pm$ & $35 \pm$ & $97.56 \pm$ & $35.34 \pm$ & $97.01 \pm$ & $33.45 \pm$ & $95.66 \pm$ & $32.68 \pm$ & $94.84 \pm$ & $26.02 \pm$ & $94.03 \pm$ & $23.84 \pm$ \\
\hline & 2.51 & 1.43 & 2.53 & $0.72 * \$$ & 2.38 & $1.12^{\star \$ \$}$ & 2.17 & $0.89^{\star \$}$ & 2.12 & $0.66^{\$ \$}$ & 2.03 & $0.89 * \#$ & 2.05 & $1.02 * \#$ \\
\hline \multirow{2}{*}{2} & $98.01 \pm$ & $48.55 \pm$ & $95.76 \pm$ & $33.39 \pm$ & $97.27 \pm$ & $34.28 \pm$ & $96.34 \pm$ & $33.36 \pm$ & $95.24 \pm$ & $32.06 \pm$ & $94.41 \pm$ & $25.85 \pm$ & $93.82 \pm$ & $20.55 \pm$ \\
\hline & 2.37 & 1.22 & 2.24 & $0.5^{\star \$}$ & 2.15 & $1.06^{\star \$}$ & 2.07 & $0.94^{\star \$}$ & 1.96 & $0.7 \approx \$$ & 1.88 & $0.85 * \#$ & 1.87 & $0.65^{*} \#$ \\
\hline \multirow{2}{*}{4} & $97.87 \pm$ & $49.35 \pm$ & $95.33 \pm$ & $30.75 \pm$ & $97.04 \pm$ & $33.78 \pm$ & $96.22 \pm$ & $32.74 \pm$ & $94.75 \pm$ & $31.75 \pm$ & $93.76 \pm$ & $25.31 \pm$ & $93.48 \pm$ & $19.35 \pm$ \\
\hline & 2.45 & 1.33 & 2.12 & $0.39 \star \$$ & 2.11 & $1.12^{\star \$ \$}$ & 1.92 & $0.98^{\star \$}$ & 1.89 & $0.88^{*} \#$ & 0.98 & $0.67 * \#$ & 2.05 & $0.25^{*} \#$ \\
\hline \multirow{2}{*}{6} & $97.81 \pm$ & $48.76 \pm$ & $95.16 \pm$ & $30.7 \pm$ & $96.77 \pm$ & $33.44 \pm$ & $96.01 \pm$ & $32.21 \pm$ & $94.58 \pm$ & $29.64 \pm$ & $93.69 \pm$ & $24.22 \pm$ & $93.10 \pm$ & $15.52 \pm$ \\
\hline & 2.34 & 1.4 & 2.42 & $0.43^{* \$}$ & 1.99 & $1.28^{\star \$}$ & 1.98 & $1.1 * \$$ & 1.83 & $0.83^{*} \#$ & 2.05 & $0.88 * \#$ & 2.36 & $1.02 * \#$ \\
\hline \multirow{2}{*}{12} & $97.81 \pm$ & $48.25 \pm$ & $95.83 \pm$ & $30.66 \pm$ & $96.26 \pm$ & $33.38 \pm$ & $95.5 \mathrm{I} \pm$ & $32.36 \pm$ & $94.04 \pm$ & $28.16 \pm$ & $93.41 \pm$ & $23.84 \pm$ & $92.78 \pm$ & $12.36 \pm$ \\
\hline & 2.5 & 1.52 & 2.13 & $0.44^{\star \$}$ & 2.04 & $|.3|^{\star \$}$ & 1.88 & $1.23 * \$$ & 1.89 & $0.84 * \#$ & 2.15 & I.24*\# & 1.87 & $0.95^{*} \#$ \\
\hline
\end{tabular}

${ }^{*}=\mathrm{p}<0.000 \mathrm{I} ; *=\mathrm{p}<0.00$ I (When IR groups were compared with DDW+IR); $\#=\mathrm{p}<0.002 ; \$=\mathrm{p}<0.05$ (When IR groups are compared with SIR); No symbols=Nonsignificant. SIR, Sham-irradiation (OGy); IR, Irradiation; DOX, doxorubicin hydrochloride; BCL, berberine; DDW, double distilled water

Table 2 Alteration in the radiation-induced DNA strand breaks in HeLa cells treated with $8 \mu g / m l$ berberine chloride before exposure to different doses of $\gamma$ - radiation

\begin{tabular}{|c|c|c|c|c|c|c|c|c|c|c|c|c|}
\hline \multirow{4}{*}{$\begin{array}{l}\text { Exposure } \\
\text { Dose } \\
(G y)\end{array}$} & \multicolumn{12}{|c|}{ Undamaged DNA (\%) \pm SEM } \\
\hline & \multicolumn{12}{|c|}{ Post-Irradiation Time (h) } \\
\hline & \multicolumn{2}{|l|}{0} & \multicolumn{2}{|l|}{0.25} & \multicolumn{2}{|l|}{0.5} & \multicolumn{2}{|l|}{ I } & \multicolumn{2}{|l|}{2} & \multicolumn{2}{|l|}{4} \\
\hline & DDW+IR & BCL+IR & DDW+IR & BCL+IR & DDW+IR & BCL+IR & DDW+IR & BCL+IR & DDW+IR & BCL+IR & DDW+IR & BCL+IR \\
\hline \multirow{2}{*}{0} & $97.41 \pm$ & $95.42 \pm$ & $97.58 \pm$ & $95.45 \pm$ & $97.12 \pm$ & $94.86 \pm$ & $97.44 \pm$ & $94.35 \pm$ & $97.05 \pm$ & $94.20 \pm$ & $96.73 \pm$ & $94.09 \pm$ \\
\hline & 2.71 & 2.04 & 2.36 & 2.14 & 2.28 & 2.07 & 2.01 & 2.20 & 2.24 & $2.01 @$ & 2.08 & $2.11 @$ \\
\hline \multirow{2}{*}{0.5} & $90.12 \pm$ & $84.55 \pm$ & $90.21 \pm$ & $85.32 \pm$ & $90.43 \pm$ & $87.65 \pm$ & $90.52 \pm$ & $89.26 \pm$ & $90.31 \pm$ & $90.16 \pm$ & $90.23 \pm$ & $91.33 \pm$ \\
\hline & 2.04 & 1.86 & 1.85 & 1.56@ & 1.68 & $2.04^{@}$ & 1.67 & $2.05^{@}$ & 2.14 & $1.17^{\#}$ & 2.31 & $2.33^{\#}$ \\
\hline \multirow{2}{*}{ I } & $74.54 \pm$ & $69.72 \pm$ & $74.73 \pm$ & $70.22 \pm$ & $74.94 \pm$ & $72.34 \pm$ & $75.08 \pm$ & $74.65 \pm$ & $75.24 \pm$ & $77.37 \pm$ & $75.48 \pm$ & $79.23 \pm$ \\
\hline & 1.82 & I.38@ & 1.87 & $1.88^{\#}$ & 1.88 & $2.12^{\#}$ & 1.66 & $1.87^{\#}$ & 2.05 & $\mathrm{I} . \mathrm{I} \mathrm{I}^{\#}$ & 1.95 & $2.14^{\mathrm{a}}$ \\
\hline \multirow{2}{*}{2} & $58.73 \pm$ & $51.34 \pm$ & $58.75 \pm$ & $50.25 \pm$ & $58.81 \pm$ & $48.54 \pm$ & $58.96 \pm$ & $46.62 \pm$ & $59.11 \pm$ & $42.51 \pm$ & $59.42 \pm$ & $38.33 \pm$ \\
\hline & 1.45 & I.I@ & 2.14 & $2.03^{\#}$ & 1.66 & $2.15^{\#}$ & 2.14 & $2.14^{\#}$ & 2.36 & $1.16^{\mathrm{a}}$ & 1.88 & $1.57^{\mathrm{a}}$ \\
\hline \multirow{2}{*}{3} & $39.87 \pm$ & $29.81 \pm$ & $41.58 \pm$ & $29.52 \pm$ & $46.38 \pm$ & $28.19 \pm$ & $48.51 \pm$ & $26.08 \pm$ & $51.61 \pm$ & $24.11 \pm$ & $59.44 \pm$ & $20.66 \pm$ \\
\hline & 1.10 & $0.74 @$ & 1.05 & $0.88^{\#}$ & 1.16 & $0.6 \mathrm{I}^{\#}$ & 1.43 & $0.66^{\mathrm{a}}$ & 1.12 & $1.70^{\mathrm{a}}$ & 1.33 & $0.88^{\mathrm{a}}$ \\
\hline \multirow{2}{*}{4} & $23.25 \pm$ & $15.87 \pm$ & $24.31 \pm$ & $14.54 \pm$ & $27.55 \pm$ & $12.23 \pm$ & $29.94 \pm$ & $10.54 \pm$ & $32.14 \pm$ & $8.56 \pm$ & $35.22 \pm$ & $5.21 \pm$ \\
\hline & 0.84 & 0.64@ & 2.02 & $1.58^{\#}$ & 1.84 & $1.56^{\#}$ & 2.14 & $1.2 \mathrm{I}^{\mathrm{a}}$ & 2.34 & $1.32^{\mathrm{a}}$ & 2.01 & $1.36^{\mathrm{a}}$ \\
\hline
\end{tabular}

$\mathrm{a}=\mathrm{p}<0.000 \mathrm{I} ; \#=\mathrm{p}<0.00 \mathrm{I} ; @=\mathrm{p}<0.05$ (Comparison between DDW and BCl groups); No symbols=Non-significant BCL, berberine; DDW, double distilled water; IR, Irradiation

Citation: Jagetia GC, Rao SK. Detection of DNA strand breaks by fluroscence assisted DNA unwinding (FADU) assay in Hela cells treated with berbrine chloride before exposure to various doses of $\gamma$ - radition. Int J Mol Biol Open Access. 20 I8;3(I): I4-2I. DOI: 10.15406/ijmboa.20 I8.03.00043 


\section{Effect of BCL on strand scission factor (SSF)}

DNA damage has also been expressed as strand scission factor (SSF) for unwound DNA after alkaline treatment. Irradiation of HeLa cells to $0,0.5,1,2,3$ or $4 \mathrm{~Gy}$, caused a significant and dose dependent elevation in SSF at all post-irradiation times (Table 3). The pattern of increase of SSF was identical in BCL+irradiation group after exposure to different doses of $\gamma$-irradiation except that SSF was significantly higher in this group when compared with the DDW+irradiation group at all post-irradiation times (Figure 2). The strand scission factor however, was higher for BCL treatment at all irradiation doses when compared with DDW+irradiation group (Table 3). The strand scission factor in BCL+irradiation group was approximately 1.6 folds greater than DDW + irradiation group for $2 \mathrm{~h}$ post-irradiation for 0.5 to $3 \mathrm{~Gy}$ gamma radiation, whereas, it was 4 folds greater for $4 \mathrm{~Gy}$ irradiation (Table 3).

Table 3 Alteration in the strand scission factors (SSF) in HeLa cells treated with $8 \mu \mathrm{g} / \mathrm{ml}$ berberine chloride before exposure o different doses of $\gamma$-radiation

\begin{tabular}{|c|c|c|c|c|c|c|c|c|c|c|c|c|}
\hline \multirow{4}{*}{$\begin{array}{l}\text { Exposure } \\
\text { Dose } \\
\text { (Gy) }\end{array}$} & \multicolumn{12}{|c|}{ Strand Scission Factor (SSF) \pm SEM } \\
\hline & \multicolumn{12}{|c|}{ Post-Irradiation Time (h) } \\
\hline & 0 & & 0.25 & & 0.5 & & $\mathbf{I}$ & & 2 & & 4 & \\
\hline & DDW+IR & $B C L+I R$ & DDW+IR & BCL+IR & DDW+IR & BCL+IR & DDW+IR & BCL+IR & DDW+IR & BCL+IR & DDW+IR & BCL+IR \\
\hline 0 & $\begin{array}{l}0.01522 \pm \\
0.002\end{array}$ & $\begin{array}{l}0.01574 \pm \\
0.006\end{array}$ & - & - & - & - & - & - & - & - & - & - \\
\hline 0.5 & $\begin{array}{l}0.01799 \pm \\
0.001\end{array}$ & $\begin{array}{l}0.04896 \pm \\
0.003\end{array}$ & $\begin{array}{l}0.0188 \pm \\
0.002\end{array}$ & $\begin{array}{l}0.05132 \pm \\
0.003\end{array}$ & $\begin{array}{l}0.02014 \pm \\
0.002\end{array}$ & $\begin{array}{l}0.0543 \pm \\
0.003\end{array}$ & $\begin{array}{l}0.02052 \pm \\
0.007\end{array}$ & $\begin{array}{l}0.0593 \pm \\
0.003\end{array}$ & $\begin{array}{l}0.03498 \pm \\
0.002\end{array}$ & $\begin{array}{l}0.0656 \pm \\
0.003^{\star}\end{array}$ & $\begin{array}{l}0.03610 \pm \\
0.007\end{array}$ & $\begin{array}{l}0.0821 \pm \\
0.00^{\star}\end{array}$ \\
\hline 1 & $\begin{array}{l}0.0702 \pm \\
0.0056\end{array}$ & $\begin{array}{l}0.132 \pm \\
0.071^{*}\end{array}$ & $\begin{array}{l}0.0824 \pm \\
0.001\end{array}$ & $\begin{array}{l}0.134 \pm \\
0.008^{\circ}\end{array}$ & $\begin{array}{l}0.0854 I \pm \\
0.005\end{array}$ & $\begin{array}{l}0.138 \pm \\
0.008^{\circ}\end{array}$ & $\begin{array}{l}0.08654 \pm \\
0.008\end{array}$ & $\begin{array}{l}0.1411 \pm \\
0.00^{*}\end{array}$ & $\begin{array}{l}0.1174 \pm \\
0.0078\end{array}$ & $\begin{array}{l}0.1584 \pm \\
0.01^{\star}\end{array}$ & $\begin{array}{l}0.1210 \pm \\
0.001\end{array}$ & $\begin{array}{l}0.1635 \pm \\
0.00^{\star}\end{array}$ \\
\hline 2 & $\begin{array}{l}0.1279 \pm \\
0.0087\end{array}$ & $\begin{array}{l}0.265 \pm \\
0.016^{\circ}\end{array}$ & $\begin{array}{l}0.1354 \pm \\
0.004\end{array}$ & $\begin{array}{l}0.271 \pm \\
0.004^{\circ}\end{array}$ & $\begin{array}{l}0.1367 \pm \\
0.002\end{array}$ & $\begin{array}{l}0.282 \pm \\
0.004^{*}\end{array}$ & $\begin{array}{l}0.1417 \pm \\
0.004\end{array}$ & $\begin{array}{l}0.3108 \pm \\
0.004^{*}\end{array}$ & $\begin{array}{l}0.2209 \pm \\
0.0164\end{array}$ & $\begin{array}{l}0.3118 \pm \\
0.021^{\mathrm{a}}\end{array}$ & $\begin{array}{l}0.2213 \pm \\
0.002\end{array}$ & $\begin{array}{l}0.3254 \pm \\
0.006^{\mathrm{a}}\end{array}$ \\
\hline 3 & $\begin{array}{l}0.2056 \pm \\
0.012\end{array}$ & $\begin{array}{l}0.505 \pm \\
0.038^{*}\end{array}$ & $\begin{array}{l}0.2074 \pm \\
0.005\end{array}$ & $\begin{array}{l}0.510 \pm \\
0.012^{\circ}\end{array}$ & $\begin{array}{l}0.2081 \pm \\
0.004\end{array}$ & $\begin{array}{l}0.5158 \pm \\
0.012^{*}\end{array}$ & $\begin{array}{l}0.2121 \pm \\
0.004\end{array}$ & $\begin{array}{l}0.5854 \pm \\
0.012^{\mathrm{a}}\end{array}$ & $\begin{array}{l}0.3836 \pm \\
0.023\end{array}$ & $\begin{array}{l}0.625 \pm \\
0.034^{a}\end{array}$ & $\begin{array}{l}0.3916 \pm \\
0.003\end{array}$ & $\begin{array}{l}0.6512 \pm \\
0.001^{\mathrm{a}}\end{array}$ \\
\hline 4 & $\begin{array}{l}0.2578 \pm \\
0.015\end{array}$ & $\begin{array}{l}0.775 \pm \\
0.052^{*}\end{array}$ & $\begin{array}{l}0.2615 \pm \\
0.002\end{array}$ & $\begin{array}{l}0.812 \pm \\
0.002\end{array}$ & $\begin{array}{l}0.2643 \pm \\
0.005\end{array}$ & $\begin{array}{l}0.8454 \pm \\
0.002\end{array}$ & $\begin{array}{l}0.2844 \pm \\
0.005\end{array}$ & $\begin{array}{l}0.9561 \pm \\
0.002^{\mathrm{a}}\end{array}$ & $\begin{array}{l}0.6234 \pm \\
0.035\end{array}$ & $\begin{array}{l}1.028 \pm \\
0.088^{\mathrm{a}}\end{array}$ & $\begin{array}{l}0.6422 \pm \\
0.005\end{array}$ & $\begin{array}{l}1.142 \pm \\
0.012^{\mathrm{a}}\end{array}$ \\
\hline
\end{tabular}

$\mathrm{a}=\mathrm{p}<0.00 \mathrm{I} ;=\mathrm{p}<0.004 ; *=\mathrm{p}<0.05$ (Comparison between DDW and BCL groups); No symbols=Non-significant. BCL, berberine; DDW, double distilled water: IR, Irradiation

\section{Discussion}

When ionizing radiation passes through matter, atoms are randomly ionized and excited. Because excited and ionized atoms initiate numerous chemical reactions, the passage of ionizing radiation through a cell produces abnormal alterations in the chemical properties of DNA, i.e., DNA damage..$^{41,42}$ Cells are exposed to many agents that result in damage to its DNA. These agents include ultraviolet radiation (UV-C), ionizing radiation ( $\gamma$ - and X-rays), reactive oxygen radicals, environmental chemicals, and therapeutic chemicals. The faithful repair of DNA double-strand breaks (DSBs) is probably one of the most critical tasks for a cell in order to maintain its genomic integrity since these lesions may lead to chromosome breaks or rearrangements, mutations and subsequently the cell death. ${ }^{5,43,44}$ DNA damage is most likely responsible for initiating the harmful biological effects of ionizing radiation, and it is reasonable to assume that the repair of DNA damage has a significant influence on the expression of DNA damage. Ionizing radiation initially creates various types of DNA damage and competition between repair processes and processes converting potentially repairable forms of DNA damage into irreversible forms of damage affect the probability of cell survival after irradiation. ${ }^{45-47}$ Most naturally occurring DSB(s), particularly the medically relevant are produced by ionizing radiation and that of some chemotherapeutic agents result from oxidative processes. The predominant repair pathway for repairing DNA DSB induced by ionizing radiation is non-homologous end joining (NHEJ). ${ }^{46,48}$ The optimal repair conditions vary depending upon the chemical structure of the DSB end being rejoined. ${ }^{49}$ In addition to DSB(s) induced by $\gamma$-radiation, the nucleotides proximal to the DSB site may have also become damaged and the extent of such damage limits the repair by NHEJ pathway. ${ }^{50,51}$

The treatment of HeLa cells with $8 \mu \mathrm{g} / \mathrm{ml}$ BCL before exposure to 2-4Gy resulted in a continuous elevation in damaged DNA up to $4 \mathrm{~h}$ post-irradiation and $95 \%$ of the DNA was damaged after 4 Gy exposure in BCL + irradiation group as majority of the DNA damage could not be repaired. This may be the reason for enhanced radiosensitivity of HeLa cells in our earlier study. ${ }^{38}$ The sensitivity of FADU method is comparable to alkaline elution method of measurement of DNA strand breaks. ${ }^{52}$ Fluorometric analysis of DNA unwinding (FADU assay), first reported by Birnboim and Jevcak to detect X-rayinduced DNA damage ${ }^{40}$ It is a fast and reliable technique to detect single-strand DNA breaks as an index of DNA damage induced by genotoxic agents. The fluorescent dye selectively binds to the double stranded DNA in the presence of single stranded DNA whose short duplex regions are destabilized by alkali. DNA lesions other than In situ strand breaks will not affect the rate of strand separation or be labile in alkali. ${ }^{53}$ Our experimental data indicate that the FADU method is highly sensitive to analyze the DNA strand breaks induced by $\gamma$-radiation and the same has been reported by other authors, where they have been able to detect the damage produced by 1-10cGy to $3 \mathrm{~Gy}$ of ionizing radiation. ${ }^{10,54-57} \mathrm{BCL}$ has increased the strand breaks induced by $\gamma$-rays up to $4 \mathrm{~h}$ in the present study.

The exact mechanism of induction of DNA damage by BCL is not known. The increased DNA damage may not be ascribed to a single mechanism and several putative mechanisms may be responsible for 
the increase in the induction of radiation-induced DNA damage by $\mathrm{BCL}$. Ionizing radiation interacts with cellular genome by induction of $\mathrm{OH}$ free radicals and the presence of $\mathrm{BCL}$ might have further increased the free radicals as it is known to induce free radicals. ${ }^{58-60} \mathrm{BCL}$ has been found to be effective in inducing DNA damage by increasing the strand breaks without allowing repair of DNA damage by any of the repair pathways including NHEJ since the DNA damage constantly increased up to $4 \mathrm{~h}$ in the present study. The action of topoisomerase II on DNA leads to its relaxation before transcription and its replication of DNA by cutting one strand of DNA duplex and passing a second duplex through this transient cleavage, the "Cleavable complex" ${ }^{61-63}$ The presence of BCL may have stabilized the cleavable complex and thus increased the DNA double-strand breaks in the present study. Berberine triggers inter nucleosomal DNA fragmentation and inhibit topoisomerase I and II enzymes In vitro. ${ }^{64,65}$ The primary damage consists of the enzyme covalently bound to DNA. Drug-stabilized covalent complexes are reversible when the drug is removed, but they can be converted into irreversible damage by collision with enzymes tracking along DNA, such as DNA polymerases. Many topoisomerasetargeting drugs are superb probes for DNA repair functions, since they have been shown to be highly specific for their targets. At molecular level suppression of PARP and NF- $\kappa B$ by berberine may have played an important role in suppressing the repair of DNA strand breaks as berberine has been reported to suppress PARP and NF- $\kappa$ B activation and inhibit homologous recombination repair. ${ }^{66}$ It is clear from our study that BCL has enhanced the geotoxic effects of radiation by increasing DNA damage in the form of strand breaks.

\section{Conclusion}

Treatment of HeLa cells with BCL before exposure to different doses of $\gamma$-radiation increased radiation-induced DNA damage. The increase in the radiation-induced DNA strand breaks by BCL may be due to increased oxidative stress in the form of free radicals, inhibition of topoisomerase I and II activities. At molecular level BCL may have down modulated the transcription of PARP and NF- $\mathrm{KB}$ and suppressed both the NHEJ and homologous recombination repair, which would have increased the DNA strand breaks after combination treatment.

\section{Acknowledgements}

The authors wish to thank Prof. M.S. Vidyasagar and Dr. J. G. R. Solomon, Department of Radiotherapy and Oncology, Kasturba Medical College, Manipal, India, for providing the irradiation facilities and for help in radiation dosimetry, respectively. The financial Assistance from Indian Council of Medical Research and Council of Scientific and Industrial Research, Government of India, New Delhi to carry out the above study is gratefully acknowledged.

\section{Conflict of interest}

Author declares that there is no conflict of interest.

\section{References}

1. Ahmad SS, Duke S, Jena R, et al. Advances in Radiotherapy. BMJ 2012;345(1):e7765.

2. Hellevik T, Martinez Zubiaurre I. Radiotherapy and the tumor stroma: the importance of dose and fractionation. Front Oncol. 2014;4:1.

3. Han W, Yu KN. Ionizing radiation, DNA double strand break and mutation. Advances in Genetics Research. 2010;4:197-210.

4. Lomax ME, Folkes LK, O Neill P. Biological consequences of radiationinduced DNA damage: relevance to radiotherapy. Clinical Oncology. 2013;25(10):578-85.
5. Mavragani IV, Nikitaki Z, Souli MP, et al. Complex DNA Damage: A Route to radiation-induced genomic instability and carcinogenesis. Cancers. 2017;9(7):91.

6. Hosoya N, Miyagawa K. Targeting DNA damage response in cancer therapy. Cancer Science. 2014;105(4):370-388.

7. Nunez, MI, Guerrero MR, Lopez E, et al. DNA damage and prediction of radiation response in lymphocytes and epidermal skin human cells. Int $J$ Cancer. 1998;76(3):354-361.

8. Ikushima T, Aritomi H, Morisita J. Radioadaptive response: efficient repair of radiation-induced DNA damage in adapted cells. Mutat Res. 1996;358(2):193-198.

9. Mc Grath RA, Williams R. Reconstruction In vivo of irradiated Escherichia coli deoxyribonucleic acid; the rejoining of broken pieces. Nature. 1966;212:534-535.

10. Roos WP, Binder A, Böhm L. Determination of the initial DNA damage and residual DNA damage remaining after 12 hours of repair in eleven cell lines at low doses of irradiation. Int J Radiat Biol. 2000;76(11):1493-1500.

11. Salagovic J, Kalina I, Dubayova K. Induction of single strand DNA breaks in workers professionally exposed to polycyclic aromatic hydrocarbons. Neoplasma. 1995;42(3):115-118.

12. Celotti L, Ferraro P, Biasin MR. Detection by fluorescence analysis of DNA unwinding and unscheduled DNA synthesis of DNA damage and repair induced In vitro by direct-acting mutagens on human lymphocytes. Mutat Res. 1992;281(1):17-23.

13. Nelson BC, Wright CW, Ibuki Y, et al. Emerging metrology for high-throughput nanomaterial genotoxicology. Mutagenesis. 2016;32(1):215-532.

14. Cernáková M, Kostálová D. Antimicrobial activity of berberine-a constituent of Mahonia aquifolium. Folia Microbiol (Praha). 2002;47(4):375-378.

15. Li Z, Geng YN, Jiang JD, et al. Antioxidant and anti-inflammatory activities of berberine in the treatment of diabetes mellitus. Evid Based Compl Alternat Med. 2014;2014:289264.

16. Peng WH, Lo KL, Lee YH, et al. Berberine produces antidepressant-like effects in the forced swim test and in the tail suspension test in mice. Life Sci 2007;81(11):933-938.

17. Peng L, Kang S, Yin Z, et al. Antibacterial activity and mechanism of berberine against Streptococcus agalactiae. Int J Clin Exp Pathol. 2015;8(5): 5217-5223.

18. Singh A, Duggal S, Kaur N, et al. Berberine: Alkaloid with wide spectrum of pharmacological activities. J Nat Prod. 2010;3:64-75.

19. Hu PF, Chen WP, Tang JL, et al. Protective effects of berberine in an experimental rat osteoarthritis model. Phytoth Res. 2011;25(6):878-885.

20. Cai Z, Wang C, Yang W. Role of berberine in Alzheimer's disease. Neuropsychiart Dis Treat. 2016;12:2509-2520.

21. Chang W, Li K, Guan F, et al. Berberine pretreatment confers cardioprotection against ischemia-reperfusion injury in a rat model of type 2 diabetes. J Cardiovas Pharmacol Therapeut. 2016;21(5):486-494.

22. Zhao GL, Yu LM, Gao WL, et al. Berberine protects rat heart from ischemia/reperfusion injury via activating JAK2/STAT3 signaling and attenuating endoplasmic reticulum stress. Acta Pharmacol Sinica. 2016;37(3):354-367.

23. Yin J, Xing H, Yeb J. Efficacy of berberine in patients with type 2 diabetes mellitus. Metabolism. 2008;57(5):712-717.

24. Zhang H, Wei J, Xue R, et al. Berberine lowers blood glucose in type 2 diabetes mellitus patients through increasing insulin receptor expression. Metabolism. 2010;59(2):285-292. 
25. Dange SV, Shende SS, Rane BT, et al. An observational study of the antidiabetic activity of berberine in newly diagnosed type 2 diabetes mellitus patients. J Pharm Biomed Sci. 2016;6(3):230-233.

26. Zeng XH, Zeng XJ, Li YY. Efficacy and safety of berberine for congestive heart failure secondary to ischemic or idiopathic dilated cardiomyopathy. Am J Cardiol. 2003;92(2):173-176.

27. Hu Y, Ehli EA, Kittelsrud J, et al. Lipid-lowering effect of berberine in human subjects and rats. Phytomedicine. 2012;19(10):861-867.

28. Chang X, Wang Z, Zhang J, et al. Lipid profiling of the therapeutic effects of berberine in patients with nonalcoholic fatty liver disease. $J$ Transl Med. 2016;14:266.

29. Di Pierro F, Putignano P, Ferrara T, et al. Retrospective analysis of the effects of a highly standardized mixture of Berberis aristata, Silybum marianum, and monacolins $\mathrm{K}$ and $\mathrm{KA}$ in patients with dyslipidemia. Clin Pharmacol. 2016;9:1-7.

30. Huang M, Chen S, Liang Y, et al. The role of berberine in the multi-target treatment of senile Dementia. Curr Top Med Chem. 2016;16(8):867-873.

31. Koppen LM, Whitaker A, Rosene A, et al. Efficacy of berberine alone and in combination for the treatment of hyperlipidemia: A systematic review. J Evid Compl Altern Med. 2017;22(4):956-958.

32. Yang Y, Wang Q, Xie M, et al. Berberine exerts an anti-inflammatory role in ocular Behcet's disease. Mol Med Rep. 2017;15(1):97-102.

33. Jagetia GC, Baliga MS. Effect of Alstonia scholaris in enhancing the anticancer activity of berberine in the Ehrlich ascites carcinoma bearing mice. J Med Food. 2004;7(2):235-244.

34. Sun Y, Xun K, Wang Y, et al. A systematic review of the anticancer properties of berberine, a natural product from Chinese herbs. Anticancer Drug. 2009;20(9):757-769.

35. Pierpaoli E, Damiani E, Orlando F, et al. Antiangiogenic and antitumor activities of berberine derivative NAX014 compound in a transgenic murine model of HER2/neu-positive mammary carcinoma Carcinogenesis. 2015;36(10):1169-1179.

36. Zou K, Li Z, Zhang Y, et al. Advances in the study of berberine and its derivatives: a focus on anti-inflammatory and anti-tumor effects in the digestive system. Acta Pharmacol Sinica. 2017;38(2):157-167.

37. Jagetia GC, Rao SK. Determination of the Antineoplastic Activity of Berberine Isolated from Tinospora cordifolia in Swiss Albino 002 Mice Transplanted with Ehrlich Ascites Carcinoma. J Tumor Med Prev. 2017;1(3):555-561.

38. Jagetia GC, Rao SK. Modulation of radioresponse by an Isoquinoline alkaloid berberine in cultured hela cells exposed to different doses of $\gamma$ -radiation. Curr Trends Biomed Eng Biosci. 2017;8(1):555-726.

39. Karnam KC, Ellutla M, Bodduluru LN, et al. Preventive effect of berberine against DMBA-induced breast cancer in female Sprague Dawley rats. Biomed Pharmacother. 2017;92:207-214.

40. Birnboim HC, Jevcak JJ. Fluorometric method for rapid detection of DNA strand breaks in human white blood cells produced by low doses of radiation. Cancer Res.1981;41(5):1889-1892.

41. Dizdaroglu M, Jaruga P. Mechanisms of free radical-induced damage to DNA. Free Rad Res. 2012;46(4):382-419.

42. Spitz DR, Hauer Jensen M. Ionizing radiation-induced responses: Where free radical chemistry meets redox biology and medicine. Antioxid. Redox Signal. 2014;20(9):1407-1409.

43. Rothkamm K, Löbrich M. Misrepair of radiation-induced DNA doublestrand breaks and its relevance for tumorigenesis and cancer treatment (Review). Int J Oncol. 2002;21(2):433-440.
44. Durante M, Bedford JS, Chen DJ, et al. From DNA damage to chromosome aberrations: joining the break. Mutat Res. 2013;756(1):5-13.

45. Tobias CA. The Repair-Misrepair Model in Radiobiology: Comparison to Other Models. Radiat Res Suppl. 1985;8:77-95.

46. Vignard J, Mirey G, Salles B. Ionizing-radiation induced DNA doublestrand breaks: a direct and indirect lighting up. Radiother Oncol. 2013;108(3):362-369.

47. Mehta A, Haber JE. Sources of DNA double-strand breaks and models of recombinational DNA repair. Cold Spring Harbor Perspect Biol. 2014;6(9):a016428.

48. Chapman JR, Taylor MR, Boulton SJ. Playing the end game: DNA doublestrand break repair pathway choice. Mol Cell. 2012;47(4):497-510.

49. Pastwa E, Neumann RD, Mezhevaya K, et al. Repair of radiationinduced DNA double strand breaks is dependent upon radiation quality and the structural complexity of double-strand breaks. Radiat Res. 2003;159(2):251-261.

50. Utsumi H, Elkind MM. Requirement for repair of DNA double-strand breaks by homologous recombination in split-dose recovery. Radiat Res. 2001;155(5):680-686.

51. Datta K, Purkayastha S, Neumann RD, et al. Base damage immediately upstream from double-strand break ends is a more severe impediment to nonhomologous end joining than blocked 3'-termini. Radiat Res. 2010;175(1):97-112.

52. Baumstark Khan C, Aufderheide E, Rink H. X-ray induced DNA strand break induction and rejoining in cultured bovine lens epithelial cells. Opthalmic Res. 1992;24(4):220-227.

53. Rydberg B. DNA unwinding in alkali applied to the study of DNA replication in mammalian cells. FEBS Lett. 1975;54(2):196-200.

54. Rydberg B. Detection of induced DNA strand breaks with improved sensitivity in human cells. Radiation Research. 1980;81(3):492-495.

55. Rogers KR, Apostol A, Madsen SJ, et al. Detection of low dose radiation induced DNA damage using temperature differential fluorescence assay. Anal Chem. 1999;71(19):4423-4426.

56. Jagetia GC, Venkatesha VA. Mangiferin protects human peripheral blood lymphocytes against $\gamma$-radiation-induced DNA strand breaks: a fluorescence analysis of DNA unwinding assay. Nut Res. 2006;26(6):303-311.

57. Moreno Villanueva M, Pfeiffer R, Sindlinger T, et al. A modified and automated version of the 'Fluorimetric Detection of Alkaline DNA Unwinding'method to quantify formation and repair of DNA strand breaks. BMC Biotechnol. 2009;9(1):39.

58. O’Neill P, Wardman P. Radiation chemistry comes before radiation biology. Int J Radiat Biol. 2009;85(1):9-25.

59. Azzam EI, Jay Gerin JP, Pain D. Ionizing radiation-induced metabolic oxidative stress and prolonged cell injury. Cancer Lett. 2012;327(1-2):48-60.

60. Pereira CV, Machado NG, Oliveira PJ. Mechanisms of berberine (natural yellow 18)-induced mitochondrial dysfunction: Interaction with the adenine nucleotide translocator. Toxicol Sci. 2008;105(2):408-417.

61. Tewey KM, Rowe TC, Yang L, et al. Adriamycin-induced DNA damage mediated by mammalian DNA topoisomerase II. Science. 1984;226(4673):466-468.

62. Pommier Y, Schwartz RE, Zwelling LA, et al. Effects of DNA intercalating agents on topoisomerase II induced DNA strand cleavage in isolated mammalian cell nuclei. Biochemistry. 1985;24(23):6406-6410. 
63. Chen SH, Chan NL, Hsieh TS. New mechanistic and functional insights into DNA topoisomerases. Ann Rev Biochem. 2013;82:139-70.

64. Makhey D, Gatto B, Yu C, et al. Protoberberine alkaloids and related compounds as dual inhibitors of mammalian topoisomerase I and II. Med Chem Res. 1995;5:1-12.

65. Qin Y, Pang JY, Chen WH, et al. Inhibition of DNA Topoisomerase I by Natural and Synthetic Mono-and Dimeric Protoberberine Alkaloids. Chem Biodiv. 2007;4(3):481-487.
66. Hou D, Xu G, Zhang C, et al. Berberine induces oxidative DNA damage and impairs homologous recombination repair in ovarian cancer cells to confer increased sensitivity to PARP inhibition. Cell Death Dis. 2017;8(10):e3070. 